\title{
Characterization of Non-amyloidogenic G101S Transthyretin
}

\author{
Yuriko Wakita, ${ }^{a, b, \#}$ Takashi Sato, ${ }^{c, \#}$ Keisuke Chosa, ${ }^{a}$ Mary Ann Suico, ${ }^{a}$ Ryoko Sasaki, ${ }^{a}$ \\ Shingo Kawano, ${ }^{c}$ Nami Hashimoto, ${ }^{a}$ Yuriko Teranishi, ${ }^{a}$ Yoshiki Imai, ${ }^{a, b}$ Hiroshi Morioka, ${ }^{c}$ \\ Tsuyoshi Shuto, ${ }^{*}, a$ and Hirofumi Kai ${ }^{*, a}$ \\ ${ }^{a}$ Department of Molecular Medicine, Graduate School of Pharmaceutical Sciences; 5-1 Oe-Honmachi, Chuo- \\ ku, Kumamoto 862-0973, Japan: ${ }^{b}$ Program for Leading Graduate Schools "HIGO (Health life science: \\ Interdisciplinary and Glocal Oriented) Program"; 5-1 Oe-Honmachi, Chuo-ku, Kumamoto 862-0973, Japan: and \\ ${ }^{c}$ Department of Analytical and Biophysical Chemistry, Kumamoto University; 5-1 Oe-Honmachi, Chuo-ku, \\ Kumamoto 862-0973, Japan.
}

Received December 20, 2017; accepted December 26, 2017

Transthyretin (TTR) is a tetrameric beta-sheet-rich protein that is important in the plasma transport of thyroxine and retinol. Mutations in the TTR gene cause TTR tetramer protein to dissociate to monomer, which is the rate-limiting step in familial amyloid polyneuropathy. Amyloidogenicity of individual TTR variants depends on the types of mutation that induce significant changes in biophysical, biochemical and/or biological properties. G101S TTR variant was previously identified in a Japanese male without amyloidotic symptom, and was considered as a non-amyloidogenic TTR variant. However, little is known about G101S TTR. Here, we found slight but possibly important biophysical differences between wild-type (WT) and G101S TTR. G101S TTR had slower rate of tetramer dissociation and lower propensity for amyloid fibril formation, especially at mild low pH (4.2 and 4.5), and was likely to have strong hydrophobic interaction among TTR monomers, suggesting relatively higher stability of G101S TTR compared with WT TTR. Cycloheximide (CHX)-based assay in HEK293 cells revealed that intracellular G101S TTR expression level was lower, but extracellular expression was higher than WT TTR, implying enhanced secretion efficiency of G101S TTR protein compared with WT TTR. Moreover, we found that STT3B-dependent posttranslational $\mathrm{N}$-glycosylation at $\mathrm{N98}$ residue occurred in G101S TTR but not in other TTR variants, possibly due to amino acid alterations that increase $\mathrm{N}$-glycosylation preference or accelerate rigid structure formation susceptible to $N$-glycosylation. Taken together, our study characterizes G101S TTR as a stable and $N$-glycosylable TTR, which may be linked to its non-amyloidogenic characteristic.

Key words transthyretin (TTR); kinetic stability; thermodynamic stability; $N$-glycosylation; STT3B; amyloidogenicity

Transthyretin (TTR), also known as prealbumin, is a $55-\mathrm{kDa}$ transport protein for the thyroid hormone thyroxine (T4) and retinol-binding protein (RBP) bound to vitamin A (retinol). ${ }^{1)}$ TTR protein has a dimer of dimers quaternary structure (homotetramer) that is synthesized mainly in the liver and also in choroid plexus and retinal pigment epithelium. ${ }^{2)}$ Certain mutations in the TTR gene destabilize TTR tetramer structure, and induce rate-limiting dissociation of native tetramer, followed by partial unfolding of the monomer to an aggregation-competent conformation. Due to its beta-sheetrich structure, unfolded TTR monomers have mutual interaction that drives amyloid fibril formation and cause hereditary TTR amyloidosis (ATTR-amyloidosis). ${ }^{3)}$ Some TTR variants are known to show non-amyloidogenic phenotype, some of which have trans-suppressive effect that renders TTR extraordinarily stable towards tetramer dissociation in compound heterozygotes carrying the pathogenic mutation. ${ }^{4)}$ To date, over 120 different TTR variants have been reported, although onset of the disease, tissue selectivity and severity generally depend on the type of mutation that directly associates with protein stability. The protein stability of TTR is dictated by the combination of kinetic stability, defined by the rate of tetramer dissociation, and thermodynamic stability, defined by the linked tetramer $\leftrightarrow$ folded monomer $\leftrightarrow$ unfolded mono- mer transition. ${ }^{5)}$ Notably, importance of TTR modification, especially posttranslational $N$-glycosylation at Asparagine 98 (N98), in the determination of TTR intracellular fate is now being considered as one of the crucial factors that could explain amyloidogenicity in patients with ATTR-amyloidosis. ${ }^{6}$ In this connection, understanding the extracellular and intracellular characteristics of TTR variants has been the research focus to develop tailored therapeutic approaches for ATTR-

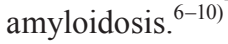

The Gly101Ser (G101S) variant was first described in 1998, detected by electrospray ionization/mass spectrometry. ${ }^{11)}$ Because G101S TTR variant was found in the blood sample of Japanese male without amyloidotic symptom, G101S TTR is considered as a non-amyloidogenic TTR variant. ${ }^{11)}$ However, little is known about the features of G101S TTR protein. Moreover, because G101 is close to N98 residue, it may be reasonable to speculate that serine substitution at G101 residue in TTR affects $N$-glycosylation status of TTR. In the present study, we compared in vitro and in vivo characteristics of wild-type (WT) and G101S TTR proteins. We observed relatively higher stability and secretion efficiency of G101S TTR compared to WT TTR. Furthermore, we found that STT3Bdependent posttranslational $N$-glycosylation at N98 residue occurs in G101S TTR protein.

\footnotetext{
\# These authors contributed equally to this work.

* To whom correspondence should be addressed. e-mail: tshuto@gpo.kumamoto-u.ac.jp; hirokai@gpo.kumamoto-u.ac.jp
} 


\section{MATERIALS AND METHODS}

Plasmid Constructs and Antibody Human WT TTR cDNA sequence was generated and inserted into the XhoI and BamHI sites of the pEF6 vector for expression from the EF- $1 \alpha$ promoter as previously described. ${ }^{7}$ D18G TTR was constructed as previously described. ${ }^{7)}$ Other TTR variants were generated utilizing the QuikChange II site-directed mutagenesis kit procedure from Stratagene (La Jolla, CA, U.S.A.) using WT TTR DNA as template. Primers used for the mutagenesis are shown in Table 1. Antibodies used are as follows: rabbit anti-human prealbumin antibody (A0002) was obtained from Dako Cytomation; anti- $\gamma$ tubulin (C-20) antibodies was obtained from Santa Cruz Biotechnology (Santa Cruz, CA, U.S.A.); mouse anti-KDEL antibody (ADI-SPA-827) was obtained from Enzo Life Sciences (Farmingdale, NY, U.S.A.); mouse anti-GM130 (\#610822) was obtained from BD Biosciences (Bedford, MA, U.S.A.); horseradish peroxidase (HRP)conjugated anti-rabbit, anti-mouse and anti-goat antibodies were from Jackson ImmunoResearch Laboratories (Westgrove, PA, U.S.A.); anti-mouse Alexa Fluor488- or anti-rabbit Alexa Fluor546-conjugated antibodies were from Molecular Probes (Eugene, OR, U.S.A.).

Expression and Purification of WT and G101S TTR Proteins All the recombinant TTRs were prepared in an Escherichia coli expression system. Briefly, the E. coli strain BL-21(DE3) was transformed with the expression plasmid for TTR. WT and G101S TTRs were expressed as fusion protein with GB1, a solubilization tag, hexahistidine-tag and following HRV3C protease digestion site. The recombinant TTRs were purified using a nickel-nitrilotriacetic acid (Ni-NTA) resin (Qiagen) and subsequently digested with HRV3C protease to remove the tag. Non-tagged recombinant TTRs were purified by gel filtration chromatography on a Superdex 75 column with $50 \mathrm{~mm} N$-(2-hydroxyethyl)piperazine- $N$ '-2-ethanesulfonic acid (HEPES) buffer (pH 7.4) and $150 \mathrm{~mm} \mathrm{NaCl}$.

Sodium Dodecyl Sulfate-Polyacrylamide Gel Electrophoresis (SDS-PAGE) and Semi-native PAGE for Recombinant TTR Proteins For SDS-PAGE, protein samples

Table 1. List of Primer for Mutagenesis

\begin{tabular}{ll}
\hline \hline Primer name & \multicolumn{1}{c}{ Target sequence } \\
\hline A97G TTR & Fw 5'-GTATTCACAGGCAACGACTCCGGCC-3' \\
& Rv 5'-GGCCGGAGTCGTTGCCTGTGAATAC-3' \\
A97S TTR & Fw 5'-GGTATTCACAAGCAACGACTCCGGCCC-3' \\
& Rv 5'-GGGCCGGAGTCGTTGCTTGTGAATACC-3' \\
G101S, N98Q TTR & Fw 5'-GGTATTCACAGCCCAGGACTCCGGCCCCCG-3' \\
& Rv 5'-CGGGGGCCGGAGTCCTGGGCTGTGAATACC-3' \\
P102R TTR & Fw 5'-CGACTCCGGCCGCCGCCGCTAC-3' \\
& Rv 5'-GTAGCGGCGGCGGCCGGAGTCG-3' \\
G101Q TTR & Fw 5'-CAGCCAACGACTCCCAGCCCCGCCGCTACAC-3' \\
& Rv 5'-GTGTAGCGGCGGGGCTGGGAGTCGTTGGCTG-3' \\
G101A TTR & Fw 5'-CAGCCAACGACTCCGCCCCCCGCCGCTACAC-3' \\
& Rv 5'-GTGTAGCGGCGGGGGGCGGAGTCGTTGGCTG-3' \\
G101M TTR & Fw 5'-CAGCCAACGACTCCATGCCCCGCCGCTACAC-3' \\
& Rv 5'-GTGTAGCGGCGGGGCATGGAGTCGTTGGCTG-3' \\
G101W TTR & Fw 5'-CAGCCAACGACTCCTGGCCCCGCCGCTACAC-3' \\
& Rv 5'-GTGTAGCGGCGGGGCCAGGAGTCGTTGGCTG-3' \\
R103S TTR & Fw 5'-CAACGACTCCGGCCCCAGCCGCTACACCATTGCC-3' \\
& Rv 5'-GGCAATGGTGTAGCGGCTGGGGCCGGAGTCGTTG-3' \\
R104C TTR & Fw 5'-GACTCCGGCCCCCGCTGCTACACCATTGCCGCC-3' \\
& Rv 5'-GGCGGCAATGGTGTAGCAGCGGGGGCCGGAGTC-3' \\
\hline
\end{tabular}

were suspended in SDS sample buffer $(0.05 \mathrm{M}$ Tris- $\mathrm{HCl}(\mathrm{pH}$ 6.8), $2 \%$ SDS, $0.82 \mathrm{M} \beta$-mercaptoethanol, $10 \%$ glycerol and $0.01 \%$ bromophenol blue (BPB)) and boiled for $10 \mathrm{~min}$. For semi-native PAGE, protein samples were suspended in native sample buffer $(0.05 \mathrm{M}$ Tris- $\mathrm{HCl}(\mathrm{pH} 6.8), 10 \%$ glycerol and $0.10 \%$ BPB) and left unboiled before loading onto gels. Both samples were analyzed by $16 \%$ polyacrylamide gel containing $0.1 \%$ SDS. Theoretically, SDS-PAGE-derived TTR band represents monomer state; while semi-native PAGE condition is considered as non-reduced and non-boiled condition, and the band observed represents tetramer state of TTR protein. ${ }^{12)}$

Urea Denaturation Measured by Tryptophan Fluorescence Denaturation reaction mixtures containing varying concentrations of urea $(0-8 \mathrm{M})$ and TTR $(0.1 \mathrm{mg} / \mathrm{mL})$ in $50 \mathrm{~mm}$ HEPES buffer ( $\mathrm{pH} 7.4$ ) and $150 \mathrm{~mm} \mathrm{NaCl}$ were incubated for $96 \mathrm{~h}$ at $4^{\circ} \mathrm{C}$. The denaturation kinetics was measured at 3 or $6 \mathrm{M}$ urea. Fluorescence emission spectra were recorded on a Hitachi F-4500 spectrofluorometer (Hitachi, Japan) at $25^{\circ} \mathrm{C}$ using a $1-\mathrm{cm}$ path length quartz cell. The excitation wavelength was $295 \mathrm{~nm}$; emission was recorded over a spectrum from 310 to $380 \mathrm{~nm}$. The fluorescence ratio at $355 \mathrm{~nm}$ and $335 \mathrm{~nm}$ was used as a structural probe as described previously. ${ }^{13)}$ The midpoint of urea denaturation $\left(C_{\mathrm{m}}\right)$ of the TTR was determined by fitting the data to a two-state model as previously described. ${ }^{14)}$

Circular Dichroism (CD) Spectroscopy CD spectra were measured using a JASCO J-820 (Nihon Bunko, Japan). Far-UV CD experiments (200-250 nm) were carried out with $0.1 \mathrm{mg} / \mathrm{mL}$ TTR samples using a $1-\mathrm{mm}$ quartz cuvette. Near-UV experiments $(250-310 \mathrm{~nm})$ were performed with $0.5 \mathrm{mg} / \mathrm{mL}$ TTR samples using a $10-\mathrm{mm}$ quartz cuvette.

Acid-Mediated TTR Amyloid Fibril Formation TTR amyloid fibril formation assay were carried out by mixing $0.4 \mathrm{mg} / \mathrm{mL}$ TTR in $50 \mathrm{~mm}$ HEPES buffer ( $\mathrm{pH} 7.4)$ and $150 \mathrm{~mm}$ $\mathrm{NaCl}$ with equal amount of acetate buffer $(200 \mathrm{~mm}$ sodium acetate buffer, $\mathrm{pH} 3.8-4.5,50 \mathrm{~mm} \mathrm{NaCl}$ and $2 \mathrm{~mm}$ ethylenediaminetetraacetic acid (EDTA)). The TTR solutions were incubated without agitation at $37^{\circ} \mathrm{C}$ for $3 \mathrm{~d}$. TTR aggregates were monitored by Thioflavin $\mathrm{T}$ (ThT) binding assay. ThT binding assay was performed as described previously. ${ }^{15}$ Fluorescence emission spectra were obtained with excitation and emission wavelengths of 430 and $485 \mathrm{~nm}$, respectively. Fluorescence measurements were performed with a Hitachi F-4500 spectrofluorometer (Hitachi).

Cell Culture and Transfection HEK293 cells were cultured in Dulbecco's modified Eagle's medium supplemented with $10 \%$ fetal bovine serum (FBS). HeLa cells were cultured in minimal essential medium supplemented with $10 \%$ FBS. Cells were maintained at $37^{\circ} \mathrm{C}$ in a humidified atmosphere of $5 \% \mathrm{CO}_{2}$ and $95 \%$ air. Transient transfections of TTR constructs were performed with TransIT-LT-1 (Mirus Corp., Madison, WI, U.S.A.) according to the manufacturer's recommendations as described previously. ${ }^{7)}$ Specifically, TransIT-LT-1 reagent diluted with reduced serum Opti-MEM (Invitrogen, Carlsbard, CA, U.S.A.) was mixed with total DNA in a ratio of $1: 3$ (DNA/LT1) and applied to subconfluent cells. Most of the experiments were performed after $48 \mathrm{~h}$ of transfection.

Western Blotting of Intracellular and Extracellular TTR Proteins Serum-free media were centrifuged at $14000 \mathrm{rpm}$ for $15 \mathrm{~min}$ at $4^{\circ} \mathrm{C}$ to remove the debris and prepared as medium samples (extracellular forms of TTR). Cells were washed 
twice with ice-cold phosphate-buffer saline (PBS) and lysed at $4^{\circ} \mathrm{C}$ in radioimmunoprecipitation assay (RIPA) buffer $(50 \mathrm{~mm}$ Tris- $\mathrm{HCl}, 150 \mathrm{~mm} \mathrm{NaCl}, 1 \mathrm{mg} / \mathrm{mL}$ sodium deoxycholate and $1 \%$ NP-40) containing 1\% protease inhibitor cocktail (SigmaAldrich, St. Louis, MO, U.S.A.) and centrifuged at $14000 \mathrm{rpm}$ for $15 \mathrm{~min}$ at $4^{\circ} \mathrm{C}$. The supernatant was prepared as cell lysate samples (intracellular forms of TTR). Protein samples were either suspended in $2 \%$ SDS sample buffer $(0.05 \mathrm{M}$ Tris$\mathrm{HCl}(\mathrm{pH}$ 6.8), $2 \%$ SDS, $0.82 \mathrm{~m} \beta$-mercaptoethanol and $10 \%$ glycerol) and boiled for $10 \mathrm{~min}$. Samples were separated by SDS-PAGE on $15 \%$ gels and transferred onto polyvinylidene difluoride membranes (Millipore Corp., Bedford, MA, U.S.A.) and then probed with anti-human prealbumin or anti- $\gamma$ tubulin antibodies. Immunological bands were identified with HRPconjugated secondary antibody (Jackson ImmunoResearch) followed by reaction with chemiluminescence reagent SuperSignal (PIERCE, Rockford, IL, U.S.A.).

Cycloheximide Chase Experiment HEK293 cells were transfected with $0.5 \mu \mathrm{g}$ of pEF6 plasmid DNA encoding the TTRs (WT, G101S). Forty-five hours after transfection, the media were replaced with fresh media containing $200 \mu \mathrm{M} \mathrm{cy-}$ cloheximide (Sigma-Aldrich). Cells were re-incubated at $37^{\circ} \mathrm{C}$ for additional 1 or $3 \mathrm{~h}$. For 0 time point, lysates and media were recovered immediately after medium change and treatment. Proteins from cell lysate and media were analyzed by SDS-PAGE and Western blotting. Densitometric quantification of blots was performed using Image $\mathrm{J}$ software.

Immunofluorescence HeLa cells were grown on glassbottomed culture dishes and transfected with $2 \mu \mathrm{g}$ of pEF6 plasmid DNA encoding the wild type or variant TTR. Fortyeight hours after transfection, cells were fixed and stained following the procedure described previously. ${ }^{16)}$ Cells were incubated with mouse anti-KDEL, anti-GM130 or rabbit antihuman Prealbumin antibodies. For visualization, the following secondary antibodies were used: anti-mouse Alexa Fluor 488- or anti-rabbit Alexa Fluor 568-conjugated antibodies. Stained cells were observed using Keyence BZ-X700 (Keyence, Japan).

Glycosidase Digestion Cell lysates and medium were incubated with Endo H (500U; New England Biolabs, Inc., U.S.A.) and PNGase F (500 U; New England Biolabs, Inc.) for $1 \mathrm{~h}$ at $37^{\circ} \mathrm{C}$. The reaction was stopped by the addition of SDSPAGE sample buffer.

Real-Time Quantitative RT-PCR Total RNA was isolated from cells using RNAiso plus (TaKaRa, Japan) according to the manufacturer's instruction. Real-time quantitative RT-PCR analyses for the indicated nucleotides and internal control 18S ribosomal RNA (rRNA) were carried out with SYBR Green Master Mix (TaKaRa) following the manufacturer's instructions. The $C_{\mathrm{t}}$ values for each gene amplification

Table 2. List of Primer Sequence for Real-Time Quantitative RT-PCR

\begin{tabular}{lc}
\hline \hline Gene & \multicolumn{1}{c}{ Target sequence } \\
\hline STT3A & Fw 5'-GTGTGGACCGTGAAGGTTCTC-3' \\
& Rv 5'-ATCCTGACCAGCCAATGTTCTG-3' \\
STT3B & Fw 5'-AATCCACCTGTGGAGGACAGC-3' \\
& Rv 5'-TGTGACCCAGGTACAGTGGAC-3' \\
18S rRNA & Fw 5'-CGGCTACCACATCCAAGGAA-3' \\
& Rv 5'-GCTGGAATTACCGCGGCT-3' \\
\hline
\end{tabular}

were normalized by subtracting the $C_{\mathrm{t}}$ value calculated for $18 \mathrm{~S}$ rRNA. The oligonucleotide primers used in the realtime quantitative PCR are as follows: human STT3A, human STT3B and human 18S rRNA (Table 2).

Small Interfering RNA (siRNA) Transfection Forty nanomolar Stealth ${ }^{\mathrm{TM}}$ RNA interference (RNAi) targeting STT3A (HSS179991), STT3B (HSS176509) and Stealth ${ }^{\mathrm{TM}}$ RNAi Low GC Duplex (negative control) (Invitrogen) were transiently transfected into HEK293 cells using Lipofectamine RNAiMAX (Invitrogen) according to the manufacturer's instructions. Cells were typically assayed 48 or $72 \mathrm{~h}$ after transfection. In the case of co-transfection of siRNA and cDNA plasmids, plasmid transfection was performed after $24 \mathrm{~h}$ of siRNA transfection, and then cells were cultured for further $48 \mathrm{~h}$.

Statistical Analysis For quantitative analysis, most of the result represents the mean \pm standard deviation (S.D.) performed in triplicate and the data were analyzed by Student's $t$-test as indicated in each figure legend.

\section{RESULTS AND DISCUSSION}

Biophysical Property of G101S TTR To determine the biophysical properties of G101S TTR protein, we first purified WT and G101S TTR proteins in E. coli expression system and investigated their structural differences using various approaches. Glycine 101 residue is located in the flexible loop connecting $F$ and $G$ (Fig. 1A). Semi-native PAGE and SDS-PAGE analyses of purified proteins confirmed that WT TTR and G101S TTR recombinant proteins exhibit similar electrophoretic mobility of tetramer and monomer forms (Figs. $1 \mathrm{~B}, \mathrm{C})$. We next determined the thermodynamic and kinetic stabilities of each TTR protein under urea-containing condition. Because urea directly denatures only monomeric TTR but not intact TTR tetramer structures, the susceptibility to urea-dependent denaturation represents thermodynamic linkage of the TTR tetramer dissociation and monomer unfolding equilibria. ${ }^{14,17)}$ The tertiary structural changes of TTR were monitored by intrinsic tryptophan fluorescence. As shown in Fig. 1D, G101S TTR was slightly more susceptible to ureadependent denaturation than WT at lower urea concentration (urea at $<4 \mathrm{M}$ ). Consistently, G101S TTR protein showed slightly lower midpoint transition value $\left(C_{\mathrm{m}}\right)$, a denaturant concentration at which both the folded and unfolded states are equally populated at equilibrium, suggesting that monomeric G101S TTR protein may have lower thermodynamic stability (Table 3 ), reflecting the linked process, which is native tetramer $\leftrightarrow$ folded monomer $\leftrightarrow$ unfolded monomer. On the other hand, under higher urea concentration condition (urea at $>4 \mathrm{M}$ ), G101S TTR was more tolerant to urea-dependent denaturation than WT (Fig. 1D). We thus measured the kinetic stability of TTR in urea concentration that corresponds to the TTR unfolding post-transition zone $(6 \mathrm{M}$ urea) to assess the rate of tetramer dissociation. The urea-unfolding rate of G101S TTR was slower than that of WT TTR (Fig. 1E). Consistent with this, the half-time for tetramer dissociation $\left(t_{1 / 2}\right)$ was longer for G101S TTR at $28.7 \mathrm{~h}$ compared to $11.5 \mathrm{~h}$ for WT TTR under $6.0 \mathrm{M}$ urea condition (Table 3 ). We also determined the urea-unfolding rate in the transition zone of TTR unfolding ( $3 \mathrm{M}$ urea). In this condition, G101S TTR was kinetically stable, although the degree of unfolding at $96 \mathrm{~h}$ incubation was 
$\mathbf{A}$

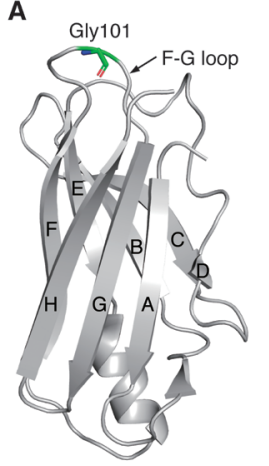

B
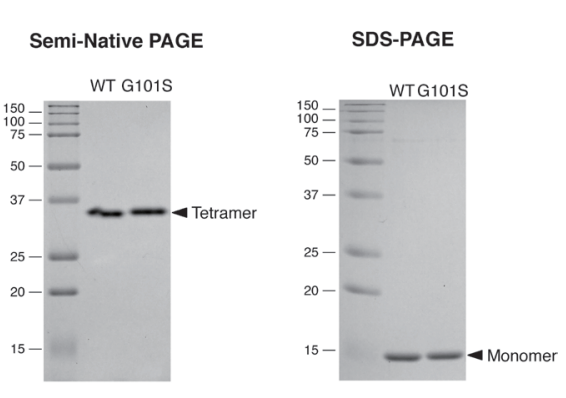

D

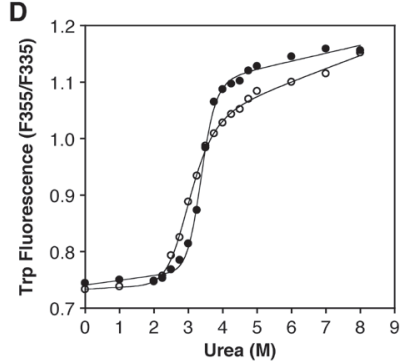

$\mathbf{F}$

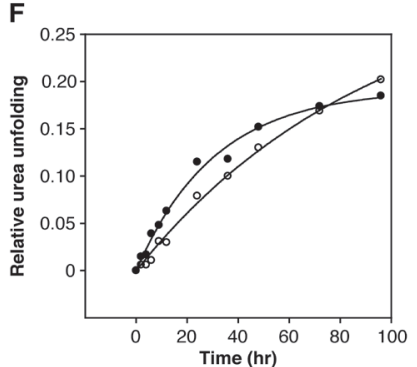

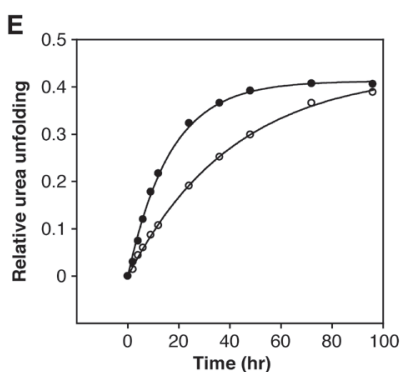

G

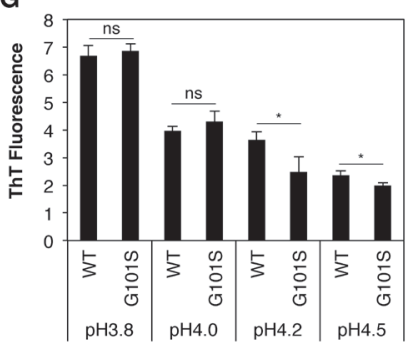

Fig. 1. Biophysical Property of G101S TTR

(A) The structure of WT TTR monomer A is shown in cartoon style. The eight $\beta$-strands in two planes are labeled DAGH and CBEF. The Gly101 residue that is shown in stick style lies in F-G-loop. Nitrogen, oxygen, and carbon are colored blue, red and green, respectively. The figure was rendered in PyMOL with the WT TTR structure (PDB 1F41). (B, C) Migration patterns of recombinant WT and G101S TTRs in semi-native PAGE (B) and SDS-PAGE (C). (D) Urea-induced denaturation curves of WT TTR (closed circles) and G101S TTR (open circles) were measured by tryptophan fluorescence. The data fitted to a two-state model. (E, F) Unfolding kinetics in $6 \mathrm{M}$ urea (E) and $3 \mathrm{~m}$ urea (F) of WT TTR (closed circles) and G101S TTR (open circles) were monitored by tryptophan fluorescence. The kinetic data fitted to single exponentials. Data shown represents the average of one experiment performed in triplicate. (G) Amyloid fibril formation of WT and G101S TTRs in various $\mathrm{pH}$ conditions was measured by thioflavin $\mathrm{T}$ (ThT) binding assay. The data are means \pm S.D. from three independent experiments. ns, not significant; $* p<0.05$; Student's $t$-test.

Table 3. Midpoint Transition Values $\left(C_{\mathrm{m}}\right)$ and Dissociation Rate of WT and G101S TTR

\begin{tabular}{lcc}
\hline \hline TTR & $C_{\mathrm{m}}(\mathrm{M})$ & $t_{1 / 2}(\mathrm{~h})$ in $6 \mathrm{M}$ urea \\
\hline WT & $3.40 \pm 0.01$ & $11.5 \pm 0.1$ \\
G101S & $3.07 \pm 0.02 *$ & $28.7 \pm 0.6^{* * *}$ \\
\hline
\end{tabular}

$C_{\mathrm{m}}$ is Midpoint transition values of urea concentration. $t_{1 / 2}$ is the half time for tetramer dissociation. All data are mean \pm S.D. from two independent experiments (Fig. 1). Unpaired Student's $t$-test: $* p<0.05, * * * p<0.001$.

higher for G101S TTR than WT TTR (Fig. 1F). These results suggest that G101S TTR has higher stability in tetramer form, but slightly lower stability in monomer form. Amyloid fibril formation of G101S TTR under milder $\mathrm{pH}$ conditions $(\mathrm{pH} 4.2$ and 4.5) was significantly lower than that of WT TTR (Fig. $1 \mathrm{G})$, further confirming that G101S TTR tetramer is relatively stable. Overall, these results suggest that G101S substitu-

A
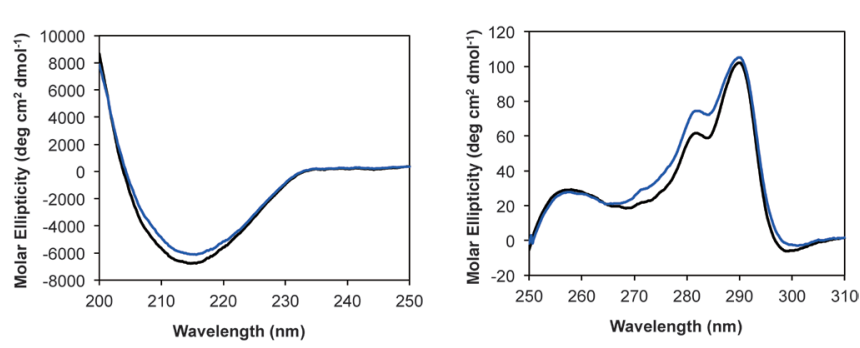

C

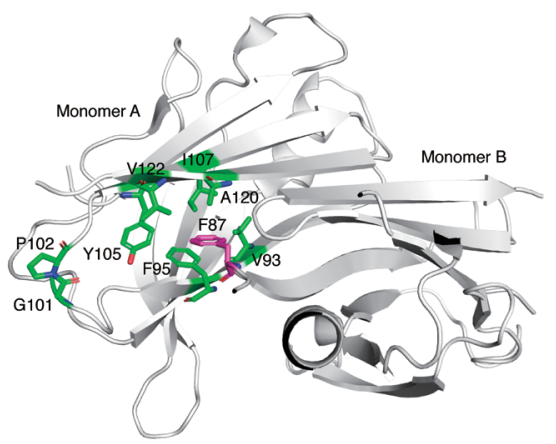

Fig. 2. Secondary and Tertiary Structures of G101S TTR

(A, B) Far UV (A) and near UV (B) CD spectra of WT TTR (black) and G101S TTR (blue). (C) Hydrophobic interaction between monomers of WT TTR. The structure of WT TTR dimer (PDB 1F41) is shown in cartoon style. The side chain of $\mathrm{F} 87$ in monomer $\mathrm{B}$, colored in magenta, projects deeply into a pocket formed by the side chain of V93, F95, Y105 I107 A120 and V112I in the monomer A, colored in green. Nitrogen, oxygen, and carbon are colored blue, red and green, respectively.

tion increases the stability of TTR tetramer, but may slightly decrease the stability of their monomeric counterparts. This biophysical character is quite unique because most of the thermodynamically less stable TTR variants show lower kinetic stability. ${ }^{18)}$ Future studies would be needed to address the trans-suppressive effect of G101S TTR.

Secondary and Tertiary Structures of G101S TTR To obtain structural insight into G101S mutant, we performed CD spectroscopy on G101S TTR at far- and near-UV regions. Both WT and G101S TTRs exhibited negative CD spectra at $216 \mathrm{~nm}$ (Fig. 2A), confirming that G101S TTR protein has proper beta-sheet-rich secondary structure. On the other hand, the $\mathrm{CD}$ spectra of G101S TTR at near-UV region, which give insight into the tertiary structure around aromatic chromophores of protein side chains (tryptophan, tyrosine, and phenylalanine), showed no significant change compared with WT TTR. But at around $270-285 \mathrm{~nm}$, which is attributable to Tyr, the spectral curve of G101S is slightly higher than that of WT TTR (Fig. 2B). These results suggest that structural fluctuations of tyrosine residues, probably in a structurally neighboring Y105 residue, may be constrained in G101S TTR. The Y105 residue forms a hydrophobic pocket together with side chain of V93, F95, Y105 I107 A120 and V112I, and is engaged in hydrophobic interaction with F87 residue in another monomer (Fig. 2C). This implies that an increase in tetramer stability in G101S TTR might be involved in strengthening the hydrophobic interaction between monomers.

The variant G101 residue is located in the loop between $\beta$-strand $\mathrm{F}$ and $\mathrm{G}$ and is followed by the Proline102 residue (Fig. 2C). Proline is a unique amino acid in its ability to adopt either cis or trans states of the backbone torsion angle $\omega$ due to its five-membered ring in the peptide backbone. ${ }^{19)}$ This 
conformational exchange can potentially serve as a precise regulator of biological function in folded proteins. ${ }^{20,21)} \mathrm{NMR}$ analysis of monomeric form of WT TTR by Lim's group showed that the cross peaks corresponding to the F-G loop were broadened by protein conformational exchange, which is due at least in part to the proline cis-trans isomerization. ${ }^{22)}$ It is believed that the cis and trans geometries of the X-proline imide bond (where $\mathrm{X}$ is any amino acid) depend on the type of amino acid in X. Compared with glycine that has a single hydrogen atom as its side chain, serine has a larger side chain, and the substitution of serine for glycine presumably shifts from the cis or trans mixed state to mainly the trans state. We speculate that this conformational change may contrib- ute to the tertiary structural change around tyrosine residues observed in near-UV CD spectroscopy and might contribute to the strengthening of the hydrophobic interaction between monomers, which is involved in Y105 residue (Fig. 2B). The structural analysis by NMR or X-ray crystallography would be needed to determine the detail of conformational changes by the G101S substitution, which is involved in the kinetic stabilization of TTR tetramer.

Character of G101S TTR in the Mammalian Cells Previous studies have demonstrated that a combination of thermodynamic and kinetic stabilities in vitro predicts the secretion efficiency and the fate of TTR variants in the cells. ${ }^{718)}$ From systematic biophysical analyses of G101S TTR, we hy-
A

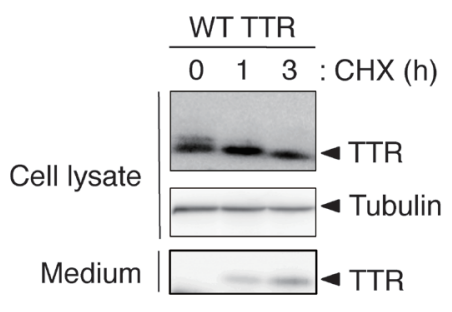

C

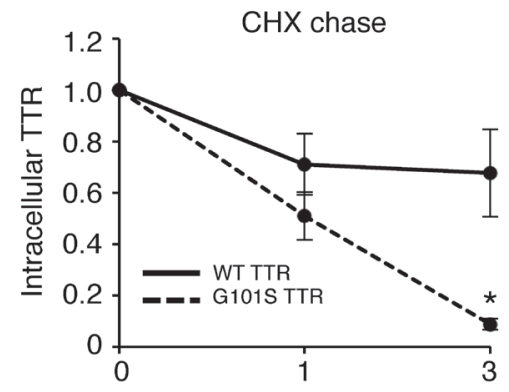

(h)

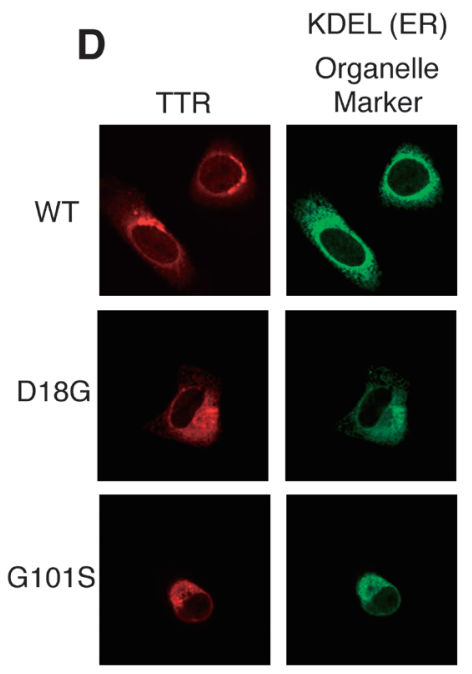

B

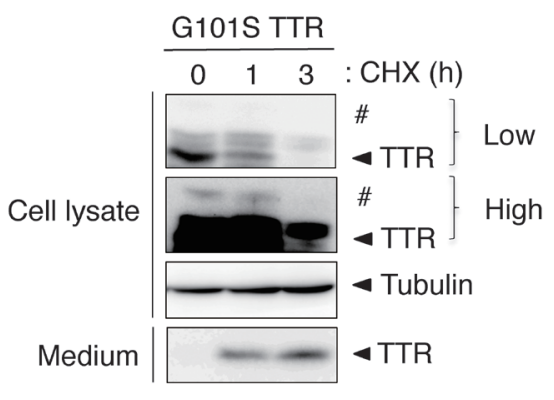

(h)
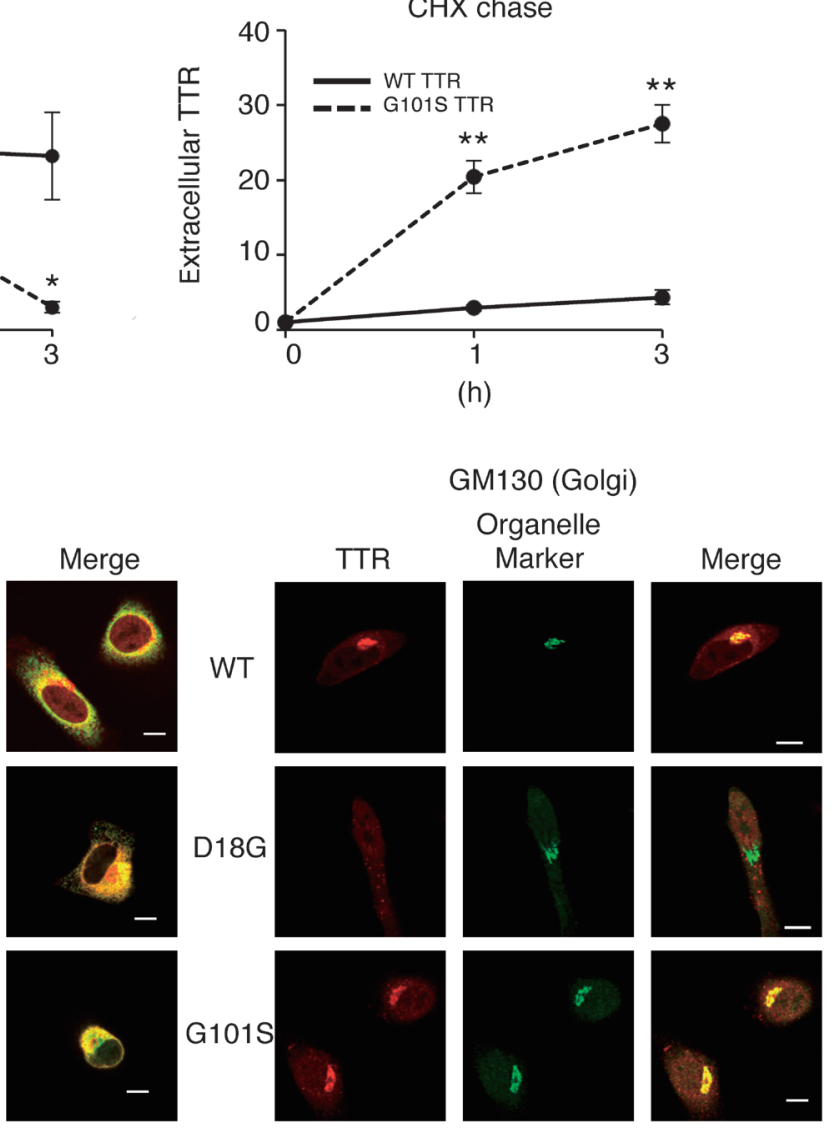

Fig. 3. Secretion and Cellular Localization of G101S TTR

(A, B) WT (A) and G101S (B) TTRs were transiently transfected in HEK293 cells. After $45 \mathrm{~h}$, media and cell lysates were collected immediately ( $0 \mathrm{~h}$ ), 1 or $3 \mathrm{~h}$ after the addition of $200 \mu \mathrm{m}$ CHX. Media and cell lysates were analyzed by Western blotting with anti-TTR and anti- $\gamma$ tubulin antibodies. \#, $N$-Glycosylated TTR. Low, high for low and high exposure, respectively. (C) Quantification of the lysate (intracellular) and media (extracellular) blots from (A) and (B). The mean and S.E. values were calculated from 3 independent experiments. ns, not significant; * $p<0.05, * * p<0.01$; Student's $t$-test. (D) HeLa cells were transfected with WT, D18G or G101S TTR. After $48 \mathrm{~h}$, cells were fixed, permeabilized and immunostained with anti-human TTR, anti-KDEL and anti-GM130 antibodies and visualized with Alexa Fluor 568-conjugated (for TTR) and Alexa Fluor 488-conjugated (for organelle markers) secondary antibodies. Scale bars, $10 \mu \mathrm{m}$. 
pothesized that the feature could influence the protein quality control of G101S TTR in the cells. Therefore, we investigated the expression level of intracellular (cell lysate) and extracellular (medium) TTR by CHX chase experiment (Figs. 3A, B). The expression level of extracellular G101S TTR at 1 and $3 \mathrm{~h}$ was higher than that of WT TTR (Fig. 3C, right panel), suggesting that G101S TTR is efficiently secreted from the cells. Consistent with the data on extracellular level of TTR, the intracellular level of G101S TTR was lower than that of
WT TTR during CHX chase period (Fig. 3C, left panel). To establish a relationship between intracellular trafficking and secretion of WT and G101S TTR, we investigated their cellular distribution by immunofluorescence staining with TTR and organelle marker (KDEL or GM130) antibodies. Compared with a non-secretory mutant D18G TTR, which is retained in the endoplasmic reticulum (ER), G101S TTR was localized in the ER and cis-Golgi compartments, as revealed by their overlap with KDEL and GM130, respectively (Fig. 3D). ${ }^{7}$ These re-
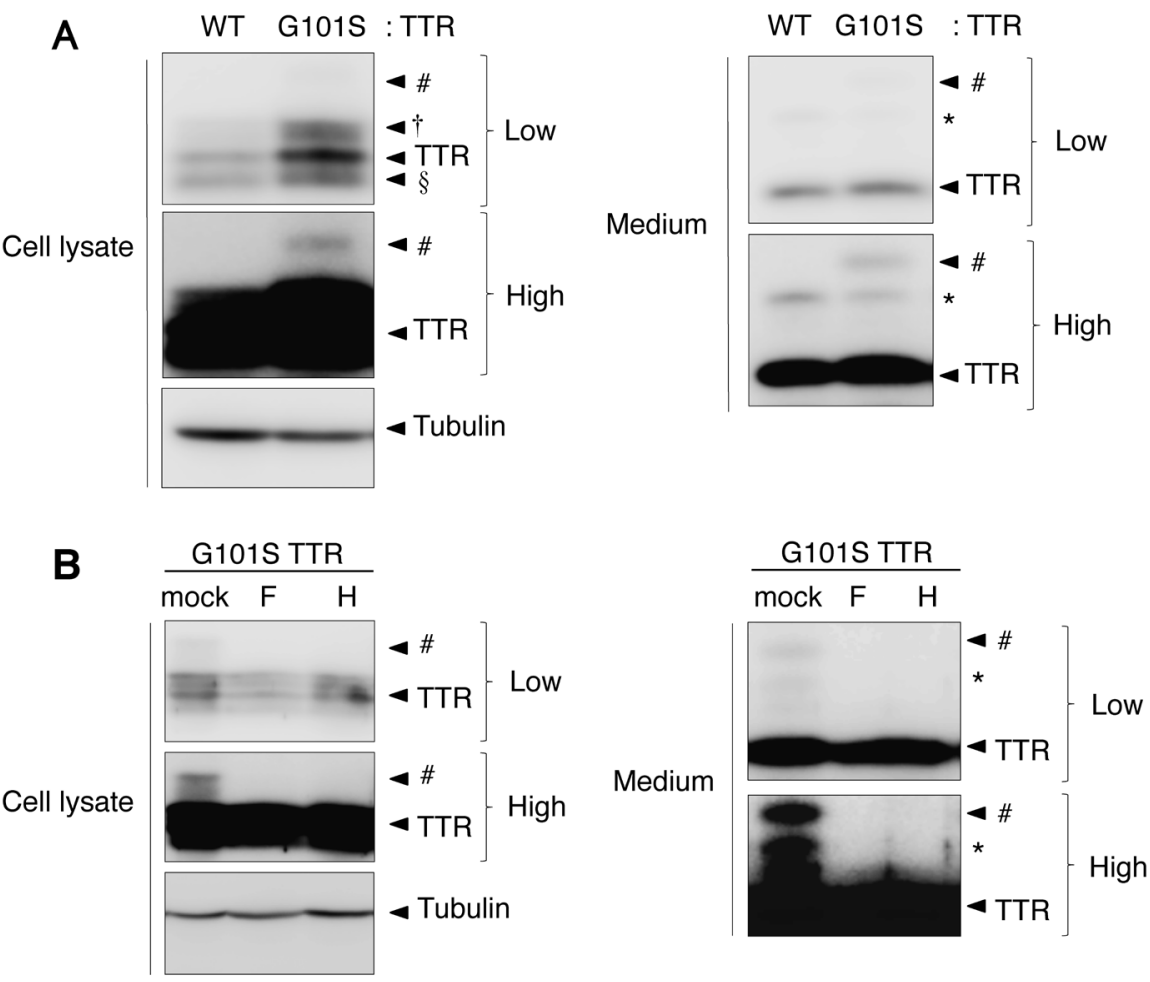

\section{C}
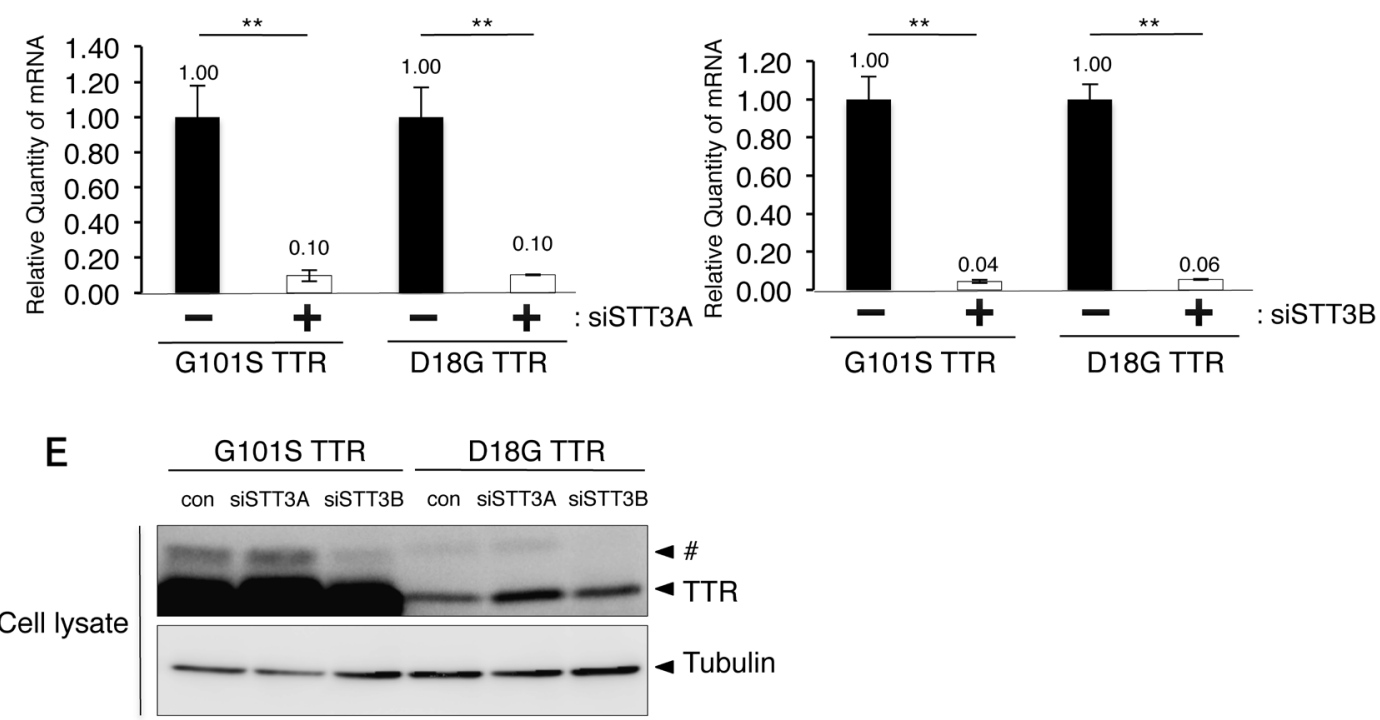

Fig. 4. HMW G101S TTR Is a N-Glycosylated Form of TTR Modified by STT3B in the OST

(A, B) HEK293 cells were transfected with WT or G101S TTR (A) and G101S TTR (B). Cell lysates and media were collected 48h post transfection and were analyzed by Western blotting with anti-TTR and anti- $\gamma$ tubulin antibodies. \#, $N$-glycosylated TTR; *, non-specific band; †, immature TTR that has an uncleaved bipartite signal sequence; $\S$, TTR that is probably cleaved by a protease. Low, High for low and high exposure, respectively. For (B), cell lysates and media were treated with PNGase F ("F") or Endo H ("H") for $1 \mathrm{~h}$ at $37^{\circ} \mathrm{C}$ before loading. (C, D) Gene expression of STT3A (C) or STT3B (D) was assessed by real-time quantitative PCR in HEK293 cells transfected with G101S TTR or D18G TTR and siCON, siSTT3A or siSTT3B. ** $p<0.01 \quad(n=3)$; Student's $t$-test. (E) HEK293 cells were transfected with G101S or D18G TTR with siCON, siSTT3A or siSTT3B. Cell lysates were collected for immunoblotting with anti-TTR and anti- $\gamma$ tubulin antibodies. 
sults indicate that G101S TTR is efficiently trafficked through the normal protein secretory pathway.

G101S TTR Is Subject to STT3B-Dependent $\mathbf{N}$-Glycosylation In the $\mathrm{CHX}$ chase data, we noticed the appearance of a high molecular weight (HMW) form of G101S TTR that was also observed in D18G TTR in our previous study. ${ }^{6}$ ) This HMW form of G101S TTR was detected in cell lysate and medium samples at high exposure of blots (Fig. 4A; High). TTR has a cryptic $N$-glycosylation consensus site (sequon),
N98-D99-S100, near the C terminus. Thus, we hypothesized that HMW-G101S TTR could be the $N$-glycosylated form of TTR. As we expected, the HMW form of G101S TTR in cell lysates and medium was susceptible to Peptide- $N$-Glycosidase F (PNGase F) (Fig. 4B). Secreted glycoproteins receive a mature, complex oligosaccharide in the medial- to trans-Golgi apparatus, which is not cleaved by Endo H. Interestingly, the intracellular and secreted PNGase F-sensitive form of G101S TTR was also susceptible to Endo H (Fig. 4B), suggesting
A

\begin{tabular}{|c|c|}
\hline \multicolumn{2}{|c|}{ Asn-X-Ser-Y } \\
\hline $\begin{array}{c}\text { Amino } \\
\text { acid (Y) }\end{array}$ & $\begin{array}{c}\text { N-glycosylation } \\
\text { efficiency (\%) }\end{array}$ \\
\hline Ser (S) & 80 \\
\hline $\operatorname{Met~(M)~}$ & 70 \\
\hline Ala (A) & 60 \\
\hline Glu (Q) & 45 \\
\hline Gly (G) & 40 \\
\hline $\operatorname{Trp}(W)$ & 35 \\
\hline
\end{tabular}

(Mellquist JL et al., Biochemistry 1998)
B

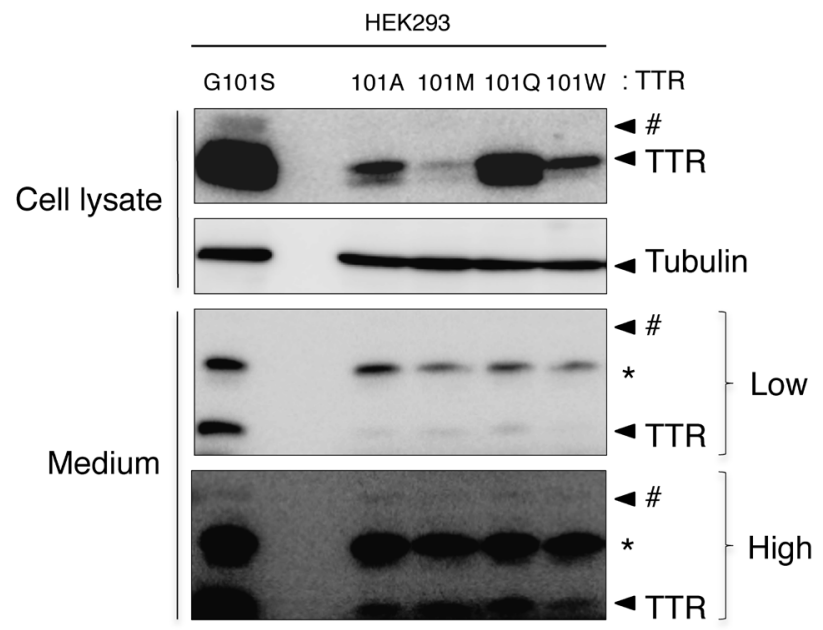

C

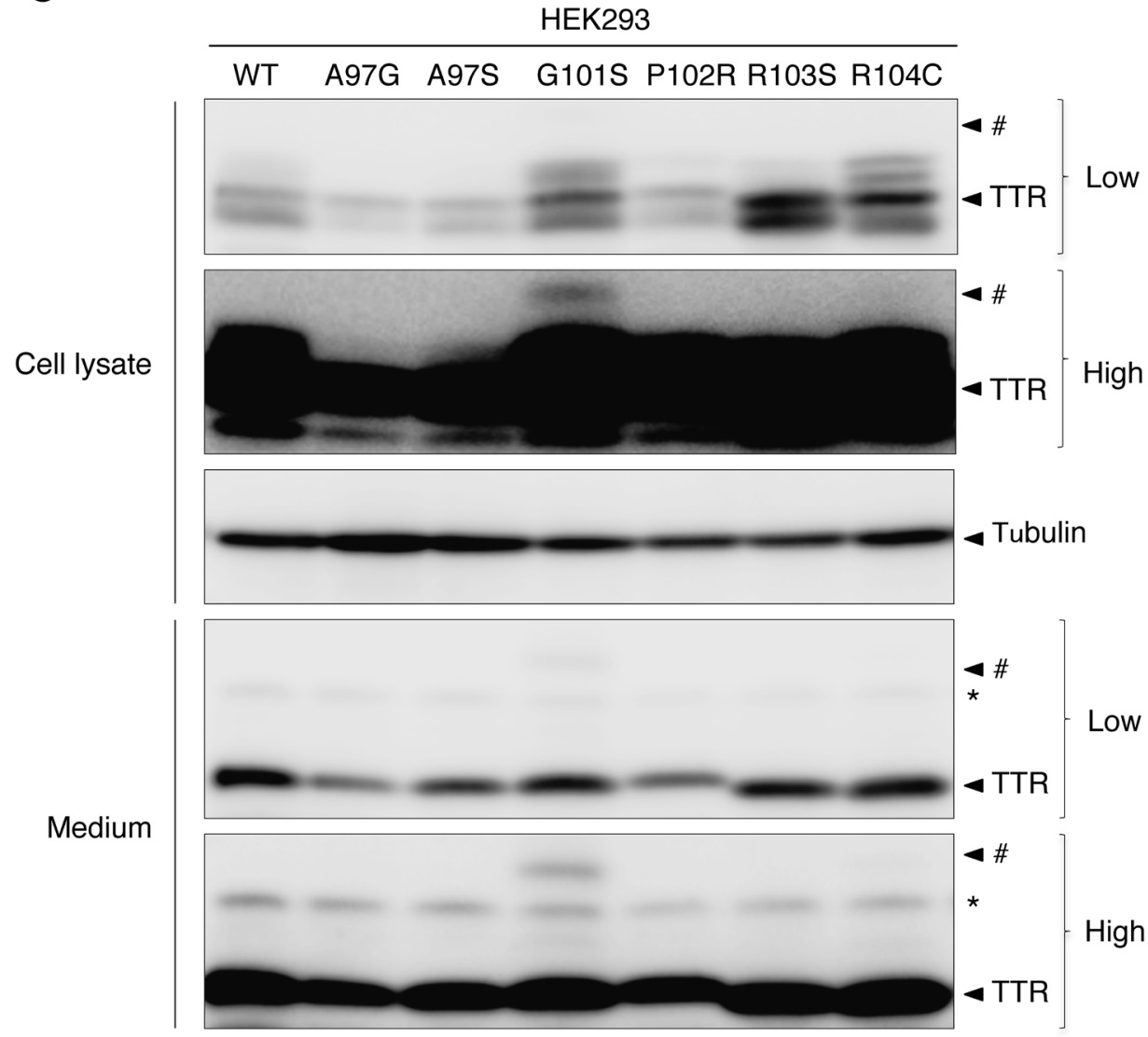

Fig. 5. Serine Substitution at G101 Residue Allows for $N$-Glycosylation

(A) Influence of the Y amino acid on the $N$-glycosylation efficiency of model glycoprotein. ${ }^{25)}$ (B, C) HEK293 cells were transfected with the indicated TTR constructs. Cell lysates and media were collected $48 \mathrm{~h}$ post transfection, and were analyzed by Western blotting. \#, $N$-glycosylated TTR; *, non-specific band. Low, high for low and high exposure, respectively. 
that $N$-glycosylated G101S TTR may be secreted through an unconventional secretory pathway, which is a poorly defined secretory route and does not require the trans-Golgi network for sorting. ${ }^{23)}$ We previously reported that normally secreted TTRs including WT and most of the mutant TTRs are unlikely to be subjected to $N$-glycosylation, while non-secreted and highly destabilized mutant such as D18G TTR is posttranslationally $N$-glycosylated by the oligosaccharyltransferase (OST) complex containing STT3B as a catalytic subunit. ${ }^{6}$ Because G101S TTR is normally secreted from the cells (Fig. $3 \mathrm{~B})$, the $N$-glycosylation modification of G101S TTR is quite a unique feature. Most of the secreted glycoproteins are cotranslationally $N$-glycosylated by OST complex equipped with the isoform STT3A. ${ }^{24)}$ To verify which OST catalytic subunit is involved in $N$-glycosylation of G101S TTR, STT3A or $S T T 3 B$ knockdown experiments using siRNA were performed. Knockdown efficiencies of STT3A and STT3B were confirmed by real-time PCR (Figs. 4C, D). Glycosylated form of G101S TTR disappeared in $S T T 3 B$ but not in STT3A knockdown, similar to the data on D18G TTR (Fig. 4E). These results suggest that G101S TTR is glycosylated by the OST complex equipped with STT3B.

Serine Substitution Next to the NDS Sequon Renders TTR Susceptible to $\mathrm{N}$-Glycosylation We previously demonstrated that excessive unfolding of TTR externalizes a cryptic $N$-glycosylation sequon and triggers posttranslational $N$-glycosylation. ${ }^{6}$ The finding that a relatively small amount of G101S TTR is $N$-glycosylated by STT3B, even though G101S TTR is a kinetically stable mutant, is an enigma. We speculate that there are other factors involved in the $N$-glycosylation of TTR that are independent of unfolding-induced sequon exposure. Mellquist and colleagues showed that the amino acid next to the $N$-glycosylation sequon (the Y position in asparagine (Asn)-X-Ser/threonine (Thr)-Y) is an important determinant of $N$-glycosylation efficiency, particularly when Ser is the hydroxy amino acid. ${ }^{25)}$ Glycosylation efficiency of rabies virus glycoprotein is approximately $40 \%$ when the amino acid at the Y position is Gly. However, this efficiency increases approximately $80 \%$ in the case of Ser at $\mathrm{Y}$ position (Fig. 5A). To gain insight into the role of Y position of the $\mathrm{N}$ glycosylation sequon in TTR, we examined the glycosylated level of TTR using a series of G101 mutants. G101S TTR but not other glycine-substituted TTRs showed a HMW band (Fig. 5B). This result suggests that the substitution of serine for glycine at Y position in TTR renders the TTR susceptible to $N$-glycosylation. Next, we investigated the glycosylation level of TTR in naturally occurring TTR variants (A97G, A97S, P102R, R103S, and R104C) that have mutation around the $\mathrm{N}$ glycosylation sequon. The $N$-glycosylation was only observed in G101S TTR but not in other variants (Fig. 5C), suggesting that the conformational change around the sequon may not be involved in $N$-glycosylation of TTR. Taken together, these results suggest that G101S mutation, located at the amino acid residue next to the $N$-glycosylation sequon, probably improves the recognition of the sequon in TTR by STT3B in OST complex.

Although TTR is a nonglycosylated secretory protein, some highly destabilized TTR variants are subject to $N$-glycosylation under specific conditions in the ER such as a crowded environment where exposure of cryptic $N$-glycosylation site could be enhanced by severe unfolding of TTR. ${ }^{6}$ In this study, we found that G101S TTR was slightly $N$-glycosylated by OST complex bearing a catalytic subunit STT3B (Fig. 4). Because the glycosylation level of G101S TTR was very low, this modification is probably a stochastic event. However, considering that this mode of glycosylation was only observed in G101S substitution (Figs. 5B, C), the $N$-glycosylation sequon, N98-D99-S100-S101, but not the original one, N98-D99-S100-G101, may be required for the efficient recognition by STT3B in OST complex. $N$-Glycosylation is known to have several functions such as stabilizing the protein structure and reducing aggregation by increasing solubility. ${ }^{26,27)}$ We previously demonstrated that $N$-glycosylation of TTR increases the solubility of aggregation-prone D18G TTR to prevent aggregation. Interestingly, Teixeira's group revealed that $N$-glycosylated TTR is detected in human plasma from V30M TTR carriers but is absent in fibrils extracted from human kidney V30M autopsy tissue. ${ }^{28)}$ These results suggested the anti-amyloidogenic role of $N$-glycosylation and the existence of a specific clearance pathway for $N$-glycosylated TTR. Therefore $N$-glycosylation of G101S TTR may contribute to its non-amyloidogenic character. Future studies would be needed to clarify the impact of $N$-glycosylation on ATTR etiopathogenesis.

\section{CONCLUSION}

In the ATTR amyloidosis, the dissociation of native tetramer and the partial monomer unfolding rendering TTR misassembly are required for the aggregates and amyloid fibril formation. To confirm whether a novel mutation shows pathogenic, non-pathogenic or trans-suppressive phenotype, biophysical characterization such as kinetic and thermodynamic stabilities of TTR in vitro is essential. Moreover, biological characterization such as the secretion and degradation levels of TTR that is regulated by protein quality control system in the ER is also important because it is involved in the disease severity and the tissue-selective amyloid deposition. In this study, we evaluated the biophysical and biological characters of G101S TTR, a TTR variant that was identified in a Japanese male. Because the patient having G101S substitution in TTR gene did not show amyloidotic symptom, G101S TTR is considered as a non-amyloidogenic TTR. We have shown here that G101S TTR is a kinetically stable and $N$-glycosylable variant, which may be linked to its non-amyloidogenic phenotype. We propose that the combination of biophysical and the biological analyses of TTR variants is important to understand the amyloidogenicity and disease etiology of a novel mutant.

Acknowledgments We thank Dr. Teruya Nakamura (Kumamoto University) and Dr. Yoshihiro Kobashigawa (Kumamoto University) for providing useful technical advice and assistance. This work was supported by the Japan Society for the Promotion Science (JSPS) KAKENHI (Grant No. JP25253012 [to HK]) and the Program for Leading Graduate Schools HIGO (Health life science: Interdisciplinary and Glocal Oriented; Ministry of Education, Culture, Sports, Science and Technology of Japan).

Conflict of Interest The authors declare no conflict of interest 


\section{REFERENCES}

1) Hamilton JA, Benson MD. Transthyretin: a review from a structural perspective. Cell Mol. Life Sci.: CMLS, 58, 1491-1521 (2001).

2) Saldaño TE, Zanotti G, Parisi G, Fernandez-Alberti S. Evaluating the effect of mutations and ligand binding on transthyretin homotetramer dynamics. PLOS ONE, 12, e0181019 (2017).

3) Hawkins PN, Ando Y, Dispenzeri A, Gonzalez-Duarte A, Adams D, Suhr OB. Evolving landscape in the management of transthyretin amyloidosis. Ann. Med., 47, 625-638 (2015).

4) Hammarström P, Schneider F, Kelly JW. Trans-suppression of misfolding in an amyloid disease. Science, 293, 2459-2462 (2001).

5) Hammarström $P$, Jiang $X$, Hurshman AR, Powers ET, Kelly JW. Sequence-dependent denaturation energetics: A major determinant in amyloid disease diversity. Proc. Natl. Acad. Sci. U.S.A., 99 (Suppl. 4), 16427-16432 (2002).

6) Sato T, Sako Y, Sho M, Momohara M, Suico MA, Shuto T, Nishitoh H, Okiyoneda T, Kokame K, Kaneko M, Taura M, Miyata M, Chosa K, Koga T, Morino-Koga S, Wada I, Kai H. STT3B-dependent posttranslational $N$-glycosylation as a surveillance system for secretory protein. Mol. Cell, 47, 99-110 (2012).

7) Sato T, Susuki S, Suico MA, Miyata M, Ando Y, Mizuguchi M, Takeuchi M, Dobashi M, Shuto T, Kai H. Endoplasmic reticulum quality control regulates the fate of transthyretin variants in the cell. EMBO J., 26, 2501-2512 (2007).

8) Henze A, Homann T, Serteser M, Can O, Sezgin O, Coskun A, Unsal I, Schweigert FJ, Ozpinar A. Post-translational modifications of transthyretin affect the triiodonine-binding potential. J. Cell. Mol. Med., 19, 359-370 (2015).

9) Zhao L, Buxbaum JN, Reixach N. Age-related oxidative modifications of transthyretin modulate its amyloidogenicity. Biochemistry, 52, 1913-1926 (2013).

10) Marcoux J, Mangione PP, Porcari R, Degiacomi MT, Verona G, Taylor GW, Giorgetti S, Raimondi S, Sanglier-Cianférani S, Benesch JLP, Cecconi C, Naqvi MM, Gillmore JD, Hawkins PN, Stoppini M, Robinson CV, Pepys MB, Bellotti V. A novel mechanoenzymatic cleavage mechanism underlies transthyretin amyloidogenesis. EMBO Mol. Med., 7, 1337-1349 (2015).

11) Kishikawa M, Nakanishi T, Miyazaki A, Hatanaka M, Shimizu A, Tamoto S, Ohsawa N, Hayashi H, Kanai M. A new nonamyloid transthyretin variant, G101S, detected by electrospray ionization/ mass spectrometry. Mutations in brief no. 201. Online. Hum. Mutat., 12, 363 (1998).

12) Robinson LZ, Reixach N. Quantification of quaternary structure stability in aggregation-prone proteins under physiological conditions: the transthyretin case. Biochemistry, 53, 6496-6510 (2014).

13) Jiang X, Smith CS, Petrassi HM, Hammarstrom P, White JT, Sacchettini JC, Kelly JW. An engineered transthyretin monomer that is nonamyloidogenic, unless it is partially denatured. Biochemistry, 40, 11442-11452 (2001).

14) Hurshman Babbes AR, Powers ET, Kelly JW. Quantification of the thermodynamically linked quaternary and tertiary structural stabilities of transthyretin and its disease-associated variants: the relationship between stability and amyloidosis. Biochemistry, 47, 6969-6984 (2008).

15) Sato $T$, Ando $Y$, Susuki S, Mikami F, Ikemizu S, Nakamura $M$, Suhr O, Anraku M, Kai T, Suico MA, Shuto T, Mizuguchi M, Yamagata Y, Kai H. Chromium(III) ion and thyroxine cooperate to stabilize the transthyretin tetramer and suppress in vitro amyloid fibril formation. FEBS Lett., 580, 491-496 (2006).

16) Okiyoneda $T$, Harada K, Takeya M, Yamahira K, Wada I, Shuto T, Suico MA, Hashimoto Y, Kai H. $\triangle$ F508 CFTR pool in the endoplasmic reticulum is increased by calnexin overexpression. Mol. Biol. Cell, 15, 563-574 (2004).

17) Groenning M, Campos RI, Fagerberg C, Rasmussen AA, Eriksen UH, Powers ET, Hammarstrom P. Thermodynamic stability and denaturation kinetics of a benign natural transthyretin mutant identified in a Danish kindred. Amyloid, 18, 35-46 (2011).

18) Sekijima Y, Wiseman RL, Matteson J, Hammarstrom P, Miller SR, Sawkar AR, Balch WE, Kelly JW. The biological and chemical basis for tissue-selective amyloid disease. Cell, 121, 73-85 (2005).

19) Andreotti AH. Native state proline isomerization: an intrinsic molecular switch. Biochemistry, 42, 9515-9524 (2003).

20) Lummis SC, Beene DL, Lee LW, Lester HA, Broadhurst RW, Dougherty DA. Cis-trans Isomerization at a proline opens the pore of a neurotransmitter-gated ion channel. Nature, 438, 248-252 (2005)

21) Sarkar P, Reichman C, Saleh T, Birge RB, Kalodimos CG. Proline cis-trans isomerization controls autoinhibition of a signaling protein. Mol. Cell, 25, 413-426 (2007).

22) Lim KH, Dyson HJ, Kelly JW, Wright PE. Localized structural fluctuations promote amyloidogenic conformations in transthyretin. J. Mol. Biol., 425, 977-988 (2013).

23) Grieve AG, Rabouille C. Golgi bypass: skirting around the heart of classical secretion. Cold Spring Harb. Perspect. Biol., 3, a005298 (2011).

24) Ruiz-Canada C, Kelleher DJ, Gilmore R. Cotranslational and posttranslational $N$-glycosylation of polypeptides by distinct mammalian OST isoforms. Cell, 136, 272-283 (2009).

25) Mellquist JL, Kasturi L, Spitalnik SL, Shakin-Eshleman SH. The amino acid following an asn-X-Ser/Thr sequon is an important determinant of N-linked core glycosylation efficiency. Biochemistry, 37, 6833-6837 (1998)

26) Culyba EK, Price JL, Hanson SR, Dhar A, Wong CH, Gruebele M, Powers ET, Kelly JW. Protein native-state stabilization by placing aromatic side chains in $N$-glycosylated reverse turns. Science, $\mathbf{3 3 1}$ 571-575 (2011).

27) Petrescu AJ, Milac AL, Petrescu SM, Dwek RA, Wormald MR. Statistical analysis of the protein environment of $N$-glycosylation sites: implications for occupancy, structure, and folding. Glycobiology, 14, 103-114 (2004).

28) Teixeira AC, Saraiva MJ. Presence of $N$-glycosylated transthyretin in plasma of V30M carriers in familial amyloidotic polyneuropathy: an escape from ERAD. J. Cell. Mol. Med., 17, 429-435 (2013). 